\title{
Sleep loss as a trigger of mood episodes in bipolar disorder: individual differences based on diagnostic subtype and gender
}

Katie Swaden Lewis, Katherine Gordon-Smith, Liz Forty, Arianna Di Florio, Nick Craddock, Lisa Jones and lan Jones

\section{Background}

Sleep loss may trigger mood episodes in people with bipolar disorder but individual differences could influence vulnerability to this trigger.

\section{Aims}

To determine whether bipolar subtype (bipolar disorder type I (BP-I) or II (BD-II)) and gender were associated with vulnerability to the sleep loss trigger.

\section{Method}

During a semi-structured interview, 3140 individuals (68\% women) with bipolar disorder (66\% BD-I) reported whether sleep loss had triggered episodes of high or low mood DSM-IV diagnosis of bipolar subtype was derived from case notes and interview data.

\section{Results}

Sleep loss triggering episodes of high mood was associated with female gender (odds ratio $(\mathrm{OR})=1.43,95 \% \mathrm{Cl} 1.17-1.75$, $P<0.001)$ and $B D-1$ subtype $(\mathrm{OR}=2.81,95 \% \mathrm{Cl} 2.26-3.50$, $P<0.001$ ). Analyses on sleep loss triggering low mood were not significant following adjustment for confounders.

\section{Conclusions}

Gender and bipolar subtype may increase vulnerability to high mood following sleep deprivation. This should be considered in situations where patients encounter sleep disruption, such as shift work and international travel.

\section{Declaration of interest}

None.

\section{Copyright and usage}

(c) The Royal College of Psychiatrists 2017. This is an open access article distributed under the terms of the creative commons Attribution (CC BY) licence.
Numerous lines of evidence suggest that sleep loss, in addition to being a core symptom and early warning sign of impending mood episodes, ${ }^{1}$ can also trigger relapse, particularly mania. ${ }^{2-4}$ However, sleep loss does not appear to be a trigger for all individuals with bipolar disorder, and those who are vulnerable to this trigger may also differ in whether sleep loss triggers mania or depression. ${ }^{5,6}$ In addition to aiding our understanding of the underlying mechanisms of bipolar illness, understanding what factors influence the relationship between sleep and mood episodes could (a) help clinicians predict which individuals are most likely to relapse following periods of sleep deprivation (for example, because of long-haul travel or shift work), and (b) inform self-management techniques such as 'e-monitoring." Two factors that may influence vulnerability to relapse following sleep loss are bipolar diagnostic subtype and gender. Bipolar disorder type I (BD-I) and type II (BD-II) are subtypes of bipolar disorder that are distinguished (according to DSM criteria) via the presence of manic (BD-I) or merely hypomanic (BD-II) episodes. ${ }^{8}$ Exploring whether there are differential reported effects of sleep disruption on mood in BD-I and BD-II is important, given that they represent two well-defined diagnostic subtypes within bipolar disorder ${ }^{8}$ with distinct clinical features and illness course. ${ }^{9}$ Gender may also influence vulnerability to mood episodes following sleep loss, as emerging evidence suggests that women are more prone to emotional dysregulation following sleep deprivation ${ }^{10-12}$ and more likely than men to experience insomnia. ${ }^{13}$ Therefore, within a large, well-characterised sample of individuals with bipolar disorder, the Bipolar Disorder Research Network (BDRN) study, we examined rates of mood episodes triggered by sleep loss according to bipolar subtype and gender.

\section{Method}

\section{Participants}

Our sample was drawn from the BDRN, a research programme that aims to identify the environmental and genetic determinants of bipolar disorders using rich phenotypic data. ${ }^{14}$ Participants were recruited from across the UK, both systematically (via National Health Service (NHS) community mental health teams) and non-systematically (via advertisements in the media and through patient support organisations, such as Bipolar UK). The study protocol was approved by an NHS research ethics committee (MREC/97/7/01) and by all participating NHS trusts and health boards. All participants provided written informed consent.

\section{Inclusion and exclusion criteria}

For inclusion into the BDRN research programme, individuals had a lifetime diagnosis of affective disorder, were at least 18 years old and of UK or Irish White ethnicity (because of the focus on genetic determinants of mood disorders). Exclusion criteria included affective disorder secondary to alcohol or substance misuse, medical illness, organic brain disorder or medication. Participants included in the current study had a DSM-IV main lifetime diagnosis of bipolar disorder (BD-I or BD-II) and had been asked at interview about sleep loss triggering mood episodes. A total of 3140 participants met these criteria.

\section{Assessments}

Trained research psychologists or psychiatrists administered all assessments and diagnostic procedures. Lifetime diagnosis was 
based on information obtained from a semi-structured interview, the Schedules for Clinical Assessment in Neuropsychiatry, ${ }^{15}$ and psychiatric case notes. Further details on derivation of diagnosis within this study are available elsewhere. ${ }^{14}$

Information on whether sleep loss had ever triggered episodes of high mood or depression was derived at interview, where participants were asked about specific past triggers of their mood episodes. Response options, in addition to sleep loss, included physical illness, medication, non-prescription drugs and alcohol. Participants' responses were coded 'yes', 'no', 'unsure' or 'not applicable' for each of the trigger options. The 'not applicable' rating was used when individuals had not experienced the relevant mood episode or trigger (for example, if a person with BD-I had never experienced a depressive episode, then questions relating to triggers of depressive episodes were rated not applicable).

\section{Statistical analysis}

All analyses were performed using SPSS version 20. Initial analyses were calculated using the chi-squared $\left(\chi^{2}\right)$ test to investigate associations between (a) bipolar subtype (BD-I v. BD-II) and (b) gender (men $v$. women), and participant reports of sleep loss triggering episodes of (hypo)mania and major depression. Initial chi-squared tests for independence included all responses to the sleep trigger questions (i.e. 'yes', 'no' and 'unsure'). Tests that revealed significant associations were followed by partitioned chi-square tests that examined only 'yes' and 'no' responses.

For analyses that were significant at the univariate level, we conducted a multivariate logistic regression analysis with bipolar subtype and gender as predictors and whether sleep loss had triggered mood episodes as the outcome. This enabled us to control for age, number of episodes of (hypo)mania or depression (depending on whether the outcome was sleep loss triggering episodes of high or low mood), and method of recruitment (systematic $v$. non-systematic). These covariates were chosen because of their potential relationship with the likelihood of reporting sleep loss as a trigger. All tests were two-tailed with a $P<0.05$ criterion for statistical significance.

\section{Results}

A total of 3140 individuals met the inclusion criteria, of whom $2075(66 \%)$ had a lifetime diagnosis of BD-I and $1065(34 \%)$ had a diagnosis of BD-II. Of our sample, $68 \%$ were women $(n=2146)$, and the mean age at interview was 46.44 years (range $18-86$, s.d. $=12.40)$.

\section{Prevalence of sleep loss triggering mood episodes in bipolar disorder}

The responses for sleep disruption and the other triggers that were specifically asked about during the interview are given in Table 1.
From the table, it can be seen that of those triggers asked about, sleep loss was the most commonly reported trigger of mood episodes in individuals with bipolar disorder. Within the total sample of 3140 individuals, 20\% (95\% CI 18.6-21.4\%, $n=627$ ) of participants reported that sleep loss had triggered episodes of mania or hypomania. For depressive episodes, $11.4 \%$ of the sample reported that sleep loss had been a trigger. A small number of participants with a diagnosis of bipolar disorder, however, will have only experienced episodes of high mood and will not, therefore, have had the opportunity to note triggers for depression. When restricting the sample to those who had experienced at least one major depressive episode $(n=3064)$, $12 \%$ (95\% CI $10.6-12.9 \%, n=359)$ reported that sleep loss had triggered episodes of depression.

\section{Differences between bipolar subtypes}

\section{Episodes of high mood}

Bipolar diagnosis (BD-I or BD-II) was associated with self-reports of sleep loss triggering episodes of high mood $\left(\chi^{2}(2)=96.189\right.$, $P<0.001)$. As shown in Fig. 1(a), individuals with a diagnosis of BD-I were more likely than those with BD-II to report that sleep loss had triggered episodes of high $\operatorname{mood}(24.7 \% \quad v$. $10.8 \%)$. Partitioned chi-square analyses comparing 'yes' and 'no' responses $(n=2952)$ revealed that BD-I participants were 2.81 times more likely than those with BD-II to report that sleep loss had triggered episodes of high $\operatorname{mood}\left(\chi^{2}(1)=90.808, P<0.001\right.$, odds ratio $(\mathrm{OR})=2.81,95 \%$ CI $2.26-3.50)$.

\section{Episodes of major depression}

Diagnostic subtype (BD-I or BD-II) was significantly associated with participants' responses on whether sleep loss had triggered episodes of depression (i.e. yes, no, unsure) $\left(\chi^{2}(2)=17.050\right.$, $P<0.001)$. As shown in Fig. 1(b), there was a trend for participants with BD-II to be more likely than those with BD-I to report that sleep loss had triggered episodes of depression $(13.6 \% v .10 .7 \%)$ but this difference did not meet criteria for statistical significance in partitioned chi-square analyses $\left(\chi^{2}(1)=3.716, P=0.054, \mathrm{OR}=1.25,95 \%\right.$ CI $\left.0.99-1.57, n=2737\right)$.

\section{Gender differences}

\section{Episodes of high mood}

As shown in Fig. 2(a), we found an association between gender and reports of sleep loss triggering episodes of (hypo)mania $\left(\chi^{2}(2)=12.739, P=0.002\right)$, with women being more likely than men to report that sleep loss had triggered episodes of high mood $(21.7 \% v .16 .3 \%)$. A partitioned chi-square analysis comparing only 'yes' and 'no' responses $(n=2952)$ showed that this difference was statistically significant $\left(\chi^{2}(1)=12.668, P<0.001\right)$ with the odds of women reporting manic or hypomanic episodes triggered

Table 1 Response frequencies of triggers of mood episodes in participants with bipolar disorder $(n=3140)$

\begin{tabular}{|c|c|c|c|c|c|}
\hline \multirow[b]{2}{*}{ Mood episode, response } & \multicolumn{5}{|c|}{ Triggers of mood episodes, $n(\%)$} \\
\hline & Sleep loss & Physical illness & Alcohol & Non-prescription drugs & Medication \\
\hline \multicolumn{6}{|l|}{ High mood } \\
\hline Yes & $627(20.0)$ & $82(2.6)$ & $231(7.4)$ & $175(5.6)$ & $114(3.6)$ \\
\hline No & $2325(74.0)$ & $2952(94.0)$ & $2706(86.2)$ & $2781(88.6)$ & $2881(91.8)$ \\
\hline Not applicable/unsure & $188(6.0)$ & $106(3.4)$ & $203(6.5)$ & $184(5.9)$ & $145(4.6)$ \\
\hline \multicolumn{6}{|l|}{ Depression } \\
\hline Yes & $359(11.4)$ & $271(8.6)$ & $242(7.7)$ & $92(2.9)$ & $79(2.5)$ \\
\hline No & $2378(75.7)$ & $2555(81.4)$ & $2526(80.4)$ & $2693(85.8)$ & $2736(87.1)$ \\
\hline Not applicable/unsure & $403(12.8)$ & $314(10.0)$ & $372(11.8)$ & $355(11.3)$ & $325(10.4)$ \\
\hline
\end{tabular}



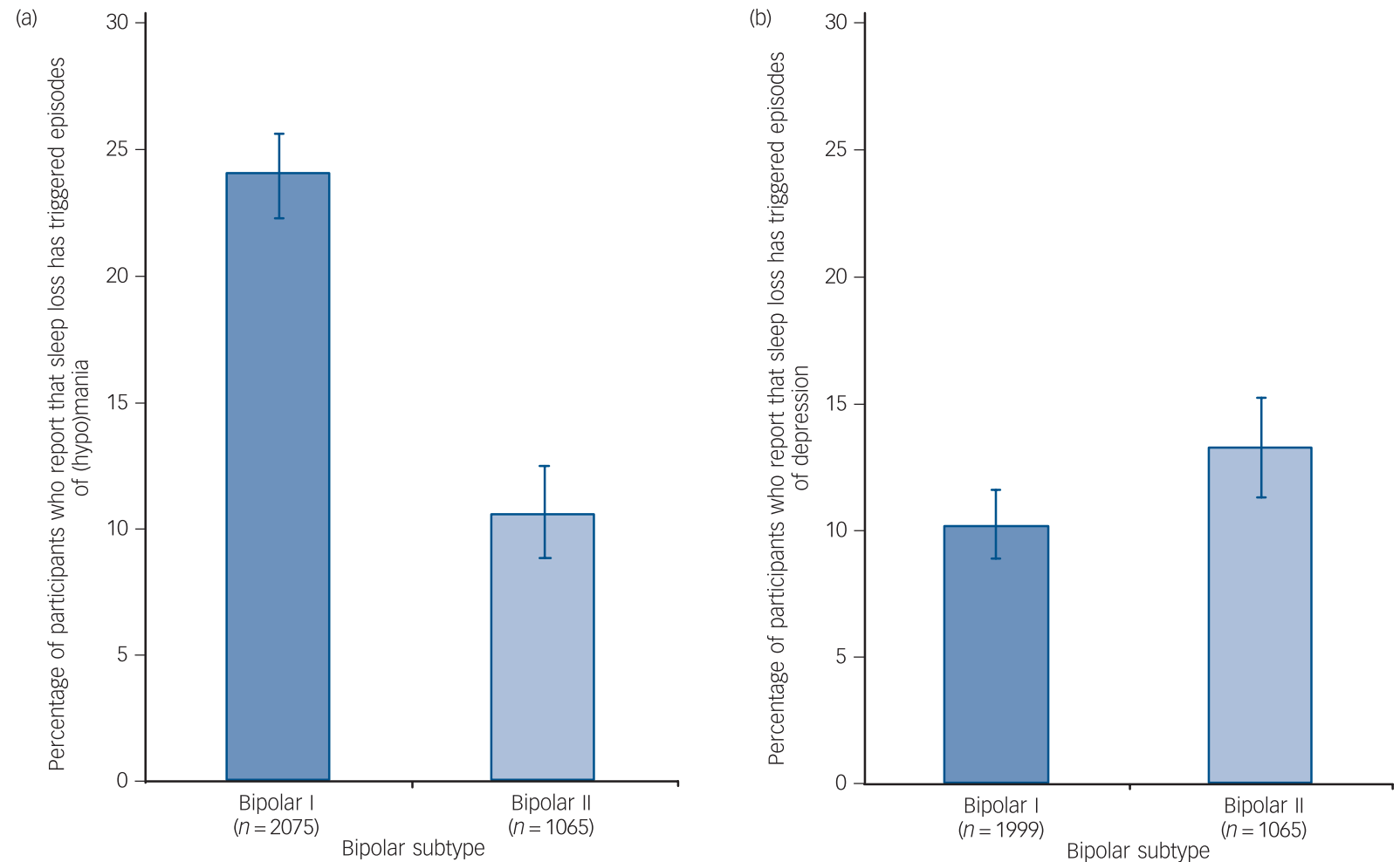

Fig. 1 Percentage of individuals with bipolar disorder who report that sleep loss has triggered episodes of (hypo)mania (a) or depression (b) split by bipolar subtype.

Error bars represent confidence intervals for point estimates.

(a)

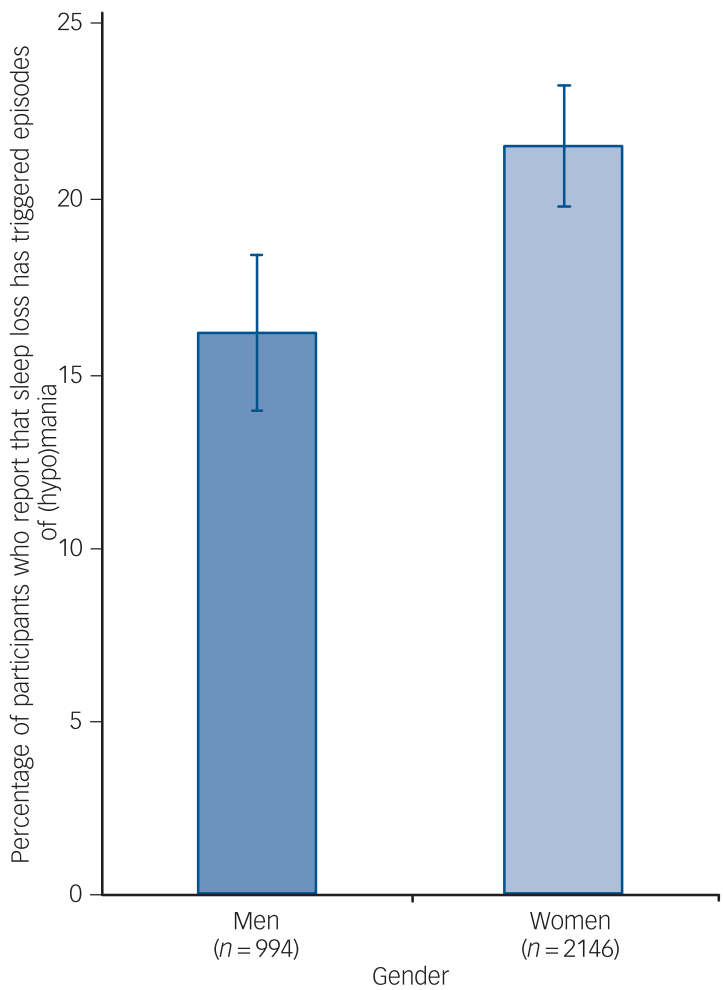

(b)

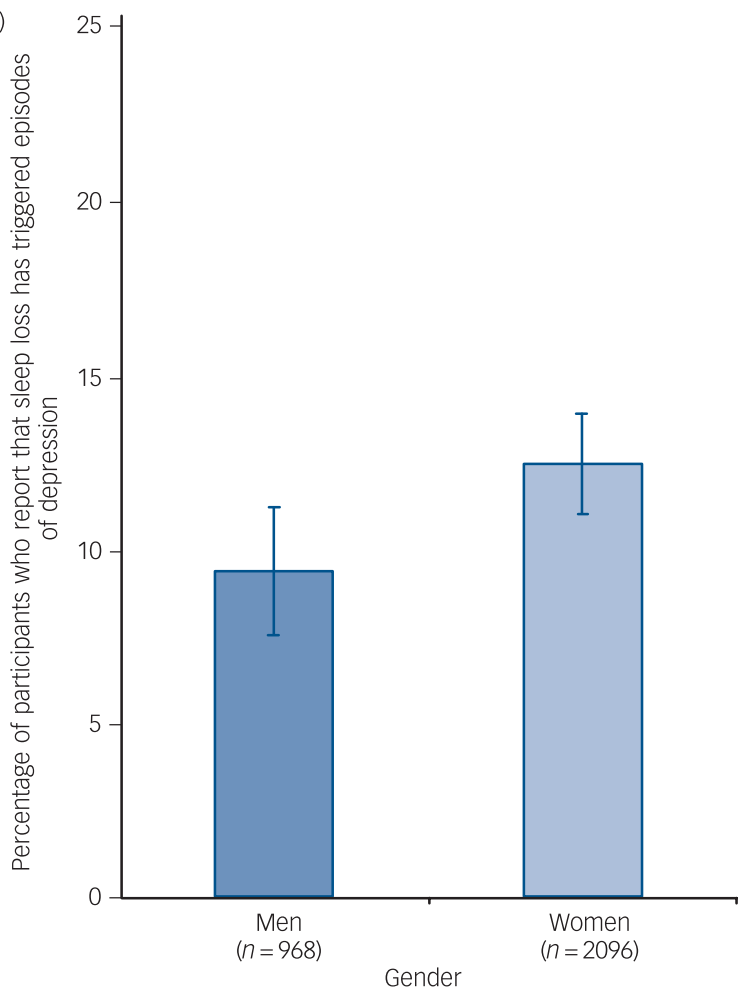

Fig. 2 Percentage of individuals with bipolar disorder who report that sleep loss has triggered episodes of (hypo)mania (a) or depression (b) split by gender.

Error bars represent confidence intervals for point estimates. 
by sleep loss being 1.43 times greater than men $(\mathrm{OR}=1.43,95 \%$ CI 1.17-1.75).

\section{Episodes of major depression}

Gender was significantly associated with self-reports of episodes of depression triggered by sleep loss $\left(\chi^{2}(2)=7.297, P=0.026\right)$. A partitioned chi-square analysis comparing only 'yes' and 'no' responses $(n=2737)$ showed that this difference was statistically significant $\left(\chi^{2}(1)=6.133, P=0.013\right)$ with women being more likely than men $(12.7 \%$ v. $9.5 \%$, respectively, see Fig. 2(b)) to report that sleep loss had triggered episodes of depression $(\mathrm{OR}=1.37,95 \%$ CI 1.07-1.77).

\section{Multivariate binary logistic regression models}

When adjusting for potential confounders, a multivariate logistic regression with (hypo)mania triggered by sleep loss as the outcome and including both bipolar diagnosis and gender as predictors found that the associations with (hypo)mania triggered by sleep loss remained significant for bipolar I diagnosis $(\mathrm{OR}=2.81,95 \%$ CI 2.23-3.53, $P<0.001)$ and female gender (OR $=1.30,95 \%$ CI 1.05-1.61, $P=0.015$. The association between female gender and episodes of depression triggered by sleep loss was not significant when controlling for potential confounders (OR $=1.29,95 \%$ CI $0.99-1.68, P=0.06)$.

\section{Discussion}

Sleep loss has been proposed as a potential trigger of mood episodes (particularly high mood) in individuals with bipolar disorder. ${ }^{2}$ Despite support from experimental research in animals ${ }^{4}$ and in healthy human populations, ${ }^{16,17}$ longitudinal studies of clinical populations have not always found that reduced sleep predicts subsequent mania in all individuals with a bipolar diagnosis. ${ }^{5,6}$ It is possible, therefore, that some individuals with bipolar disorder have increased vulnerability to experiencing high mood following sleep disruption whereas others may be unaffected or become depressed. If so, this would concord with research in healthy populations that finds individual differences in vulnerability to the neurocognitive effects of sleep loss. ${ }^{18,19}$

Our main finding was that individuals with bipolar disorder seem to differ in their tendency for sleep loss to trigger mood episodes depending on their gender and bipolar subtype. Specifically, we found that participants who were female or had a diagnosis of BD-I were more likely to report that sleep loss had triggered episodes of high mood than male participants or those with a BD-II diagnosis. In contrast, women and individuals with a BD-II diagnosis were more likely to report sleep loss triggering episodes of depression than men or those with a BD-I diagnosis. However, these differences did not meet criteria for statistical significance.

\section{Bipolar subtype}

In our sample, individuals with BD-I were significantly more likely than those with BD-II to report that sleep loss had triggered episodes of high mood, with one in four individuals with BD-I reporting this compared with around one in ten individuals with $\mathrm{BD}-\mathrm{II}$. To our knowledge, this is currently the largest study to date reporting on the prevalence of sleep loss as a trigger in individuals with bipolar disorder. Research to date has primarily focused on early symptoms of mood episodes ${ }^{1}$ or has explored the proportion of individuals with bipolar disorder who become manic or hypomanic following sleep deprivation therapy for depression.
The proportion of individuals who become manic or hypomanic in these contexts has been reported to range from $4.85 \%$ to $29 \%{ }^{20}$

Differences between bipolar subtypes in the tendency for sleep loss to trigger high mood may reflect clinical and underlying neuroanatomical (and associated functional) differences between BD-I and BD-II that have been highlighted elsewhere. ${ }^{9,21,22}$ One of the proposed mechanisms by which sleep loss affects emotion regulation is by disrupting emotion regulation systems in the brain (for example, prefrontal and limbic areas) ${ }^{16}$ and recent neuroimaging studies have found that these same neurological systems vary between individuals with BD-I and BD-II, and correspond with behavioural differences in emotion regulation. ${ }^{23}$

Alternatively, it is possible that fewer participants with BD-II reported sleep loss triggering high mood because they were generally less able to identify triggers of their episodes. However, analysis of the 'unsure' responses for this question revealed that $4.7 \%$ of participants with a BD-II diagnosis responded 'unsure' compared with $6.7 \%$ of those with BD-I $(P=0.029)$.

\section{Gender}

Our results also suggest that women may be more susceptible than men to mood dysregulation following sleep loss, with women being significantly more likely than men to report that sleep loss had triggered episodes of high mood. Our findings may have been a result of women being more likely to overreport triggers or generally being better at identifying triggers of mood episodes than men. However, when we examined responses for other triggers that participants were asked at interview (i.e. medication, antidepressants, alcohol or non-prescription drugs) we found that women did not consistently endorse triggers more than men (results available from the author on request). In addition, our analyses of 'unsure' responses found that men were not significantly more likely than women to respond 'unsure' when asked about triggers of high mood $(5.8 \% v .6 .1 \%$, respectively, $P=0.807)$ or depressive episodes ( $11.6 \% v .10 .3 \%$, respectively, $P=0.274)$.

Our findings are in agreement with previous research that has also found women to be at greater risk than men of mood disturbances following sleep loss ${ }^{11,12}$ and may partly explain why women with bipolar disorder are at high risk of experiencing mania following childbirth ${ }^{24}$ (a life event associated with sleep deprivation). Furthermore, there is evidence that women with $\mathrm{BD}-\mathrm{I}$ are more likely to become manic following childbirth compared with those with BD-II, ${ }^{25}$ which is concordant with our results for bipolar disorder subtype.

These gender differences provide interesting avenues to explore underlying mechanisms by which gender and sleep may interact to influence bipolar disorder phenotypes. For example, it has been suggested that ovarian hormones may interact with the circadian system to influence the sleep-wake cycle and responses to sleep deprivation. ${ }^{26}$

\section{Episodes of depression triggered by sleep loss}

It is possible that the association between sleep loss and mania has been attenuated in some studies by the inadvertent inclusion of individuals who, following sleep loss, are more likely to experience shifts in mood towards the opposite polarity (i.e. depression). Therefore, we examined whether bipolar subtype and/or gender were associated with an increased likelihood of reporting episodes of depression triggered by sleep loss.

First, we found some evidence that individuals with BD-II were more likely than those with BD-I to report that sleep loss had triggered episodes of depression ( $14 \% v .11 \%$, respectively) but this difference did not meet criteria for statistical significance $(P=0.054)$. Of note is that fewer participants reported sleep loss 
triggering episodes of depression $(n=359,12 \%)$ compared with high mood $(n=627,20 \%)$. In addition, comparing 'unsure' responses between participants who had experienced episodes of depression $(n=3064)$ and episodes of high mood $(n=3140)$ revealed that a great proportion of participants responded 'unsure' when asked whether sleep loss had triggered episodes of depression (10.7\%) compared with episodes of (hypo)mania $(6.0 \%)$. The reasons for this are unclear; however, Jackson et al reported that individuals with bipolar disorder found it easier to identify early symptoms of mania than depression. Therefore, if the onset of depressive episodes is less noticeable than manic episodes, it may be more difficult for individuals to identify triggers.

Second, we found that women were more likely than men to report that sleep loss had triggered episodes of depression. This concurred with previous research finding that women are more likely than men to experience depressed mood following sleep loss. ${ }^{11,12}$ Our findings may be due to women being at a greater risk of experiencing insomnia, which has been associated with subsequent depressive episodes. ${ }^{27}$ However, the association between gender and sleep loss triggering depression did not remain significant when controlling for potential confounding factors. This may be because rates of insomnia have been found to increase with age in both genders ${ }^{27}$ and our sample included a wide age range (median 46 years, range 18-86).

\section{Strengths and limitations}

The strengths of this study are that it is the first to examine sleep loss as a trigger of both manic and depressive episodes in a large sample of individuals with bipolar disorder. In contrast to previous studies, our large sample allowed us to examine whether bipolar subtype and gender affect vulnerability to sleep loss. A further strength is that participant diagnoses were derived from rich clinical data. However, our study is limited by (a) the retrospective and subjective nature of our data, and therefore (b) the inability to disentangle whether sleep loss was a trigger or prodrome of mood episodes.

First, the retrospective nature of our data means it is possible that participants over- or underestimated the role of sleep loss in triggering mood episodes. However, there is some evidence that there is a moderate correlation between objective and subjective measures of sleep in remitted patients with bipolar disorder. ${ }^{28}$ Other studies have examined this relationship using experimental methods (i.e. sleep deprivation protocols) in which sleep duration and mood are ascertained via nurse or psychiatrist observations. ${ }^{3,29,30}$ These studies provide the most convincing evidence that sleep loss can trigger relapse. However, the labourintensive nature of such studies means that the sample sizes are several orders of magnitude lower than that of our sample, (ranging from single case studies ${ }^{29}$ to 143 patients ${ }^{30}$ ). The less intensive nature of our self-report measure allowed us to collect this information in a large sample of individuals and therefore ensured that we could compare the responses of men and women, as well as bipolar subtypes. In addition, the simplicity of our measure allows for easier application to clinical practice.

Second, the bidirectional relationship between sleep and $\operatorname{mood}^{2,10,16}$ could have made it difficult for participants to judge whether sleep loss had been a trigger or prodrome of episodes (although participants had the option to respond 'unsure'). However, in addition to the studies mentioned above, multiple experimental studies have provided supporting evidence that sleep deprivation can perturb emotion regulation (for reviews, see Kahn et $a l^{16}$ and Harvey et $a l^{31}$ ) and can be a risk factor for the development of bipolar disorder in high-risk children. ${ }^{32}$ In addition, if sleep disruption is a prodrome rather than a trigger, it could nevertheless be a clinically useful warning sign of incipient mood episodes; therefore, distinguishing the characteristics of those individuals with bipolar disorder who describe a close relationship between changes in sleep and the onset of a mood episode could aid illness management.

\section{Future research}

The results of the present study should inform future research investigating associations between sleep and mood in bipolar disorder, as samples containing a mix of genders and bipolar subtypes might obfuscate associations between sleep and mood. However, our results need to be validated in longitudinal studies using objective measures of sleep such as actigraphy, as we did not have objective data on whether participants experienced sleep loss prior to mood episodes, or on the type of sleep loss that they experienced (such as total or partial sleep deprivation). Such research may also inform the rapidly expanding field of 'digital psychiatry' that aims to use technology such as smartphone applications to improve patient care. ${ }^{33}$

It is unclear why some individuals become depressed following sleep deprivation and others become manic. It is possible that other triggers associated with relapse in bipolar disorder (such as stressful or exciting life events, medication use or interpersonal conflict) may have coincided with the sleep loss that our participants reported. However, vulnerability to the negative cognitive and emotional effects of sleep loss has been studied more extensively in healthy populations, which provides further support that differential vulnerability to sleep loss is trait-like and potentially genetic (see Banks \& Dinges ${ }^{19}$ for a review). Some studies have explored this in participants with bipolar disorder, however, to date this has been limited to candidate gene studies (for example Benedetti et $a l^{34}$ ). Future research could potentially use manic or depressive responses to sleep loss as a subphenotype for genome-wide analyses or examine whether individuals at high familial risk of bipolar disorder are more prone to mood dysregulation following sleep loss.

Another intriguing avenue for further research is the relationship between sleep and pharmacology. For example, lithium is known to affect circadian rhythms, ${ }^{35}$ and there is preliminary evidence that patients with specific variants in genes that encode enzymes targeted by lithium are more likely to experience mood elevation following sleep deprivation. ${ }^{36}$

\section{Implications for clinical practice}

The findings of this study can inform self-management for bipolar disorder, particularly in light of recent attempts to engage patients with electronic self-monitoring tools, which aim to alert the individual of impending mood episodes based on fluctuations in behaviours such as sleep (e.g. Hidalgo-Mazzei et $l^{7}$ ). Adjusting these estimates based on individual differences that are likely to affect the relationship between sleep and mood will improve the accuracy of such tools.

Furthermore, our study suggests that up to one in four individuals with bipolar disorder may be at risk of an episode of high mood following sleep loss, with women and those with a BD-I diagnosis particularly at risk. Clinicians should therefore discuss the importance of this trigger with patients, including encouraging a regular sleep pattern and considering the potential impact of specific situations such as shift work and long-haul travel.

\section{Funding}

This work was supported by grants from the Wellcome Trust (078901) and the Stanley Medical Research Institute (6045240-5500000100) 


\section{Acknowledgements}

The authors would like to thank all members of the Bipolar Disorder Research Network and especially the participants who have kindly given their time to take part in ou research.

Katie Swaden Lewis, BSC, Division of Psychological Medicine and Clinical Neurosciences, MRC Centre for Neuropsychiatric Genetics and Genomics, Cardiff University, Cardiff; Katherine Gordon-Smith, PhD, Institute of Health \& Society, University of Worcester, Worcester; Liz Forty, PhD, National Centre for Mental Health, Division of Psychological Medicine and Clinical Neurosciences, MRC Centre for Neuropsychiatric Genetics and Genomics, Cardiff University, Cardiff; Arianna Di Florio, PhD, MD, Division of Psychological Medicine and Clinical Neurosciences, MRC Centre for Neuropsychiatric Genetics and Genomics, Cardiff University, Cardiff, Nick Craddock, PhD, FRCPsych, National Centre for Mental Health, Division of Psychological Medicine and Clinical Neurosciences, MRC Centre for Neuropsychiatric Genetics and Genomics, Cardiff University, Cardiff; Lisa Jones, PhD, Institute of Health \& Society, University of Worcester, Worcester; Ian Jones, PhD, MRCPsych, Neurosciences, MRC Centre for Neuropsychiatric Genetics and Genomics, Cardiff University, Cardiff, UK

Correspondence: Ian Jones, Division of Psychological Medicine and Clinica Neurosciences, MRC Centre for Neuropsychiatric Genetics and Genomics, Cardiff University, Hadyn Ellis Building, Maindy Road, Cardiff CF24 4HQ, UK. Email: JonesIR1@cf.ac.uk

First received 9 Feb 2017, final revision 31 Mar 2017, accepted 5 Apr 2017

\section{References}

1 Jackson A, Cavanagh J, Scott J. A systematic review of manic and depressive prodromes. J Affect Disord 2003; 74: 209-17.

2 Wehr TA, Sack DA, Rosenthal NE. Sleep reduction as a final common pathway in the genesis of mania. Am J Psychiatry 1987; 144: 201-4.

3 Wehr TA, Goodman FK, Wirz-Justice A, Breitmaier J, Craig C. 48-hour sleep-wake cycles in manic depressive illness. Arch Gen Psychiatry 1982; 39 559-65

4 Gessa GL, Pani L, Fadda P, Fratta W. Sleep deprivation in the rat: an anima model of mania. Eur Neuropsychopharmacol 1995; 5: 89-93.

5 Talbot LS, Stone S, Gruber J, Hairston IS, Eidelman P, Harvey AG. A test of the bidirectional association between sleep and mood in bipolar disorder and insomnia. J Abnorm Psychol 2012; 121: 39-50.

6 Perlman CA, Johnson SL, Mellman TA. The prospective impact of sleep duration on depression and mania. Bipolar Disord 2006; 8: 271-4.

7 Hidalgo-Mazzei D, Mateu A, Reinares M, Undurraga J, Bonnín CDM, Sánchez-Moreno J, et al. Self-monitoring and psychoeducation in bipolar patients with a smart-phone application (SIMPLe) project: design, development and studies protocols. BMC Psychiatry 2015; 15: 52.

8 American Psychiatric Association. Diagnostic and Statistical Manual of Mental Disorders (4th edn, revised) (DSM-IV-TR). APA, 2000.

9 Baek JH, Park DY, Choi J, Kim JS, Choi JS, Ha K, et al. Differences between bipolar I and bipolar II disorders in clinical features, comorbidity, and family history. J Affect Disord 2011; 131: 59-67.

10 Baglioni C, Spiegelhalder K, Lombardo C, Riemann D. Sleep and emotions: a focus on insomnia. Sleep Med Rev 2010; 14: 227-38.

11 Short MA, Louca M. Sleep deprivation leads to mood deficits in healthy adolescents. Sleep Med 2015; 16: 987-93.

12 Saunders EFH, Fernandez-Mendoza J, Kamali M, Assari S, Mcinnis MG. The effect of poor sleep quality on mood outcome differs between men and women: a longitudinal study of bipolar disorder. J Affect Disord 2015; 180: 90-6.

13 Mai E, Buysse DJ. Insomnia: prevalence, impact, pathogenesis, differential diagnosis, and evaluation. Sleep Med Clin 2008; 3: 167-74.

14 Jones L, Metcalf A, Gordon-Smith K, Forty L, Perry A, Lloyd J, et al. Gambling problems in bipolar disorder in the UK: prevalence and distribution. Br J Psychiatry 2015; 207: 328-33.
15 Wing JK, Babor T, Brugha T, Burke J, Cooper JE, Giel R, et al. SCAN: schedules for clinical assessment in neuropsychiatry. Arch Gen Psychiatry 1990; 47: 589-93.

16 Kahn M, Sheppes G, Sadeh A. Sleep and emotions: bidirectional links and underlying mechanisms. Int J Psychophysiol 2013; 89: 218-28.

17 Van Someren EJW, Cirelli C, Dijk D-J, Van Cauter E, Schwartz S, Chee MWL. Disrupted sleep: from molecules to cognition. J Neurosci 2015; 35: 13889-95.

18 Van Dongen HP, Baynard MD, Maislin G, Dinges DF. Systematic interindividual differences in neurobehavioral impairment from sleep loss: evidence of trait-like differential vulnerability. Sleep 2004; 27: 423-33.

19 Banks S, Dinges DF. Behavioral and physiological consequences of sleep restriction. J Clin Sleep Med 2007; 3: 519-28.

20 Plante DT, Winkelman JW. Sleep disturbance in bipolar disorder: therapeutic implications. Am J Psychiatry 2008; 162: 50-7.

21 Liu JX, Chen YS, Hsieh JC, Su TP, Yeh TC, Chen LF. Differences in white matter abnormalities between bipolar I and II disorders. J Affect Disord 2010; 127: 309-15.

22 Caseras $X$, Lawrence NS, Murphy K, Wise RG, Phillips ML. Ventral striatum activity in response to reward: differences between bipolar I and II disorders Am J Psychiatry 2013; 170: 533-41.

23 Caseras $X$, Murphy $K$, Lawrence NS, Fuentes-Claramonte $P$, Watts J, Jones DK, et al. Emotion regulation deficits in euthymic bipolar I versus bipolar II disorder: a functional and diffusion-tensor imaging study. Bipolar Disord 2015; 17: 461-70.

24 Jones I, Chandra PS, Dazzan P, Howard LM. Bipolar disorder, affective psychosis, and schizophrenia in pregnancy and the post-partum period. Lancet 2014; 384: 1789-99.

25 Di Florio A, Forty L, Gordon-Smith K, Heron J, Group $\sqcup$, Craddock N, et al. Perinatal episodes across the mood disorder spectrum. JAMA Psychiatry 2013; 70: 168-75

26 Bailey M, Silver R. Sex differences in circadian timing systems: implications for disease. Front Neuroendocrinol 2014; 35: 111-39.

27 Ohayon MM. Epidemiology of insomnia: what we know and what we still need to learn. Sleep Med Rev 2002; 6: 97-111.

28 Boudebesse C, Geoffroy PA, Bellivier F, Henry C, Folkard S, Leboyer M, et al. Correlations between objective and subjective sleep and circadian markers in remitted patients with bipolar disorder. Chronobiol Int 2014; 31: 698-704.

29 Wehr TA. Sleep-loss as a possible mediator of diverse causes of mania. Br J Psychiatry 1991; 159: 576-8.

30 Benedetti F, Riccaboni R, Locatelli C, Poletti S, Dallaspezia S, Colombo C. Rapid treatment response of suicidal symptoms to lithium, sleep deprivation, and light therapy (chronotherapeutics) in drug-resistant bipolar depression. J Clin Psychiatry 2014; 75: 133-40.

31 Harvey AG, Talbot LS, Gershon A. Sleep disturbance in bipolar disorder across the lifespan. Clin Psychol Sci Pract 2009; 16: 256-77.

32 Levenson JC, Axelson DA, Merranko J, Angulo M, Goldstein TR, Goldstein BI, et al. Differences in sleep disturbances among offspring of parents with and without bipolar disorder: association with conversion to bipolar disorder. Bipolar Disord 2015; 17: 836-48.

33 Bidargaddi N, Musiat P, Makinen V-P, Ermes M, Schrader G, Licinio J. Digital footprints: facilitating large-scale environmental psychiatric research in naturalistic settings through data from everyday technologies. $\mathrm{Mol}$ Psychiatry 2016; 22: 164-9.

34 Benedetti F, Dallaspezia S, Lorenzi C, Pirovano A, Radaelli D, Locatelli C, et al Gene-gene interaction of glycogen synthase kinase 3-beta and serotonin transporter on human antidepressant response to sleep deprivation. $J$ Affect Disord 2012; 136: 514-9.

35 McCarthy MJ, Welsh DK. Cellular circadian clocks in mood disorders. J Biol Rhythms 2012; 27: 339-52.

36 Benedetti F, Serretti A, Colombo C, Lorenzi C, Tubazio V, Smeraldi E. A glycogen synthase kinase 3-beta promoter gene single nucleotide polymorphism is associated with age at onset and response to total sleep deprivation in bipolar depression. Neurosci Lett 2004; 368: 123-6. 